Research Article Plant Genetics

\title{
Identification of histone methylation modifiers and their expression patterns during somatic embryogenesis in Hevea brasiliensis
}

\author{
Hui-Liang $\mathrm{Li}^{1}$, Dong Guo ${ }^{1}$, Jia-Hong Zhu ${ }^{1}$, Ying Wang ${ }^{1}$ and Shi-Qing Peng ${ }^{1}$ (iD \\ ${ }^{1}$ Key Laboratory of Tropical Crop Biotechnology, Ministry of Agriculture, Institute of Tropical Bioscience \\ and Biotechnology, Chinese Academy of Tropical Agricultural Sciences, Haikou, China
}

\begin{abstract}
Histone methylation plays a crucial role in various biological processes, from heterochromatin formation to transcriptional regulation. Currently, no information is available regarding histone methylation modifiers in the important rubber-producing plant Hevea brasiliensis. Here, we identified 47 histone methyltransferase (HMT) genes and 25 histone demethylase (HDM) genes as possible members of the histone methylation modifiers in the rubber tree genome. According to the structural features of HMT and HDM, the HbHMTs were classified into two groups (HbPRMs and HbSDGs), the HbHDMs have two groups (HbLSDs and HbJMJs). Expression patterns were analyzed in five different tissues and at different phases of somatic embryogenesis. HbSDG10, 21, 25, 33, HbJMJ2, 18,20 were with high expression at different phases of somatic embryogenesis. HbSDG10,14, 20, 21, 33 and HbPRMT4 were expressed highly in anther, HbSDG14, 20, 21, 22, 23, 33, 35 and HbPRMT1 HbJMJ7 and HbLSD1, 2, 3, 4 showed high expression levels in callus. HbSDG1, 7, 10, 13, 14, 18, 19, 21, 22, 23, 35, HbPRMT1, 8, HbJMJ5, 7, 11, 16, 20 and HbLSD2, 3, 4 were expressed highly in somatic embryo. HbSDG10, 21, 25, 33, HbLSD2, 3 were expressed highly in bud of regenerated plant. The analyses reveal that $H b H M T s$ and $H b H D M s$ exhibit different expression patterns at different phases during somatic embryogenesis, implying that some HbHMTs and HbHDMs play important roles during somatic embryogenesis. This study provide fundamental information for further studies on histone methylation in Hevea brasiliensis.
\end{abstract}

Keywords: : Hevea brasiliensis, histone methylation, histone methyltransferase, histone demethylase, somatic embryogenesis.

Received: May 16, 2018; Accepted: March 10, 2019.

\section{Introduction}

Histone methylation plays an essential role in maintaining genome stability and is also involved in regulating multiple cellular processes (Jenuwein and Allis, 2001; Kouzarides, 2002; Liu et al., 2010). Histone methylation can either repress or activate gene expression (Rice et al., 2003; Liu et al., 2010). The level of histone methylation is dynamically regulated by histone methyltransferases (HMTs) and histone demethylases (HDMs), respectively (Klose and Zhang, 2007; Liu et al., 2010). Histone methylation, occurring at arginine residues or lysine residues, is controlled by protein arginine methyltransferases (PRMTs) and histone lysine methyltransferases (HKMTs) (Jenuwein and Allis, 2001; Litt et al., 2009; Liu et al., 2010). HKMTs, also known as SET-domain group (SDG-like types), and PRMTs have highly conserved SET and PRMT domains involved in methyltransferase catalytic activity (Ng et al., 2007; Liu et al., 2010; Ahmad and Cao, 2012). In contrast with HMTs, HDMs remove the methyl groups from methylated lysine or arginine residues of his-

Send correspondence to Shi-Qing Peng. Institute of Tropical Bioscience and Biotechnology, Chinese Academy of Tropical Agricultural Sciences, \#4 Xueyuan Rd., Haikou 571101, China. E-mail: shqpeng@163.com. tones (Tsukada et al., 2006; Chang et al., 2007). Plant HDMs have two major types: lysine-specific demethylase1 (LSD) and jumonjiC (JmjC) domain containing proteins (Shi et al., 2004; Tsukada et al., 2006). LSD removes mono- and di-methyl groups from $\mathrm{H} 3 \mathrm{~K} 4$ residue depending on flavin adenine dinucleotide as a cofactor, while the JmjC-domain proteins require the presence of a-ketoglutarate and Fe-II cofactors (Shi et al., 2004; Klose and Zhang, 2007). JmjC proteins also remove methyl from H3R2 and H4R3 residues (Chang et al., 2007; Cho et al., 2012). In plants, histone methylation has important roles in cellular processes (Berr et al., 2011), including vegetative growth (Thorstensen et al., 2011; Liu et al., 2017), development (Cartagena et al., 2008; Grini et al., 2009; Cho et al., 2012), circadian cycle (Jones and Harmer, 2011; Lu et al., 2011), flowering process (Gan et al., 2014; Liu et al., 2015), flowering time (Liu et al., 2014, 2016), response to abiotic stress (van Dijk et al., 2010; Shen et al., 2014), disease resistance (Berr et al., 2010; Li et al., 2014), and hormone signaling (Sui et al., 2012; Li J et al., 2015; Zhao et al., 2015).

The rubber tree (Hevea brasiliensis) produces natural rubber, which is an important industrial material (Backhaus, 1985). Large scale propagation of rubber tree is 
achieved by grafting buds onto unselected seedlings (Clément-Demange et al., 2007; Hua et al., 2010). The grafted plants sometimes produce intraclonal heterogeneity for growth and productivity (Chandrashekar et al., 1997; Clément-Demange et al., 2007; Hua et al., 2010). In the early 1980s, novel plantlets, named as self-rooting juvenile clones (SRJCs), developed from internal integuments of immature fruits or anthers of $H$. brasiliensis were obtained through tissue culture (Wang et al., 1980; Carron and Enjalric, 1982). SRJCs showed better performance in growth and rubber yield than those of donor clones (DCs) (Liu et al., 1985; Yang and Mo 1994; Yuan et al., 1998; Chen et al., 2002). The molecular mechanism associated with high yield in SRJCs is not clear. Li et al. $(2014,2016)$ found that there were many differentially expressed genes between SRJCs and DCs, including genes involved in rubber biosynthesis pathway and some genes encoding epigenetic modification enzymes (Li et al., 2014, 2016). The DNA methylation level in rubber tree differs at each periods of somatic embryogenesis (Li HL et al., 2015). Epigenetic modifications may be associated with the regulation of several genes involved in natural rubber biosynthesis, resulting in the higher rubber productivity of SRJCs compared to DCs (Li et al., 2016). Histone methylation is one of the epigenetic modifications (Liu et al., 2010). Up to now, histone methylation modifiers have not been identified in Hevea brasiliensis. Using the rubber tree genome data (Rahman et al., 2013; Tang et al., 2016), we investigated histone methylation modifiers through a bioinformatics approach. Here, 47 HMT and 25 HDM genes were identified, and the expression patterns of HbMTs and $H b H D M s$ were analyzed in different tissues and at different phases of rubber tree somatic embryogenesis. This study should greatly facilitate the functional characterization of those histone methylation in the rubber producing crop species.

\section{Materials and Methods}

\section{Prediction of HMTs and HDMs in the rubber tree genome}

A local rubber tree genome database was established using the rubber tree genome data (Li et al., 2017). The HMTs and HDMs protein sequences from rice and Arabidopsis thaliana were used as query sequences (Table S1). A BLAST search was performed to detect HMTs and HDMs, with a BLAST threshold of $1 \mathrm{e}^{-5}$. To further verify the reliability of these candidate sequences, the Pfam database (http://pfam.sanger. ac.uk/search) and SMART (http://smart.embl-heidelberg.de/) were used to confirm each candidate HbHMT and HbHDM protein as a member of the HMT and HDM families.

\section{Motif detection of HbHMTs and HbHDMs}

Motif detection of HbHMTs and HbHDMs was performed in http://meme-suite.org/tools/meme (Bailey et al.,
2015). All identified motifs were further searched in the InterPro database with Inter-ProScan (Mitchell et al., 2014).

\section{Multiple sequence alignment and phylogenetic} analysis of HbHMTs and HbHDMs

Multiple sequence alignments of HbHMTs and HbHDMs were performed using ClustalX2 (http://www. clustal.org/). A phylogenetic tree of HHbMTs and HbHDMs was constructed by the Maximum Likelihood (ML) method with parameters of bootstrap (1000 replicates). Gene structures were analyzed by comparing the coding sequences of HbHMTs and HbHDMs with the corresponding genomic sequences using GSDS software (http://gsds.cbi. pku.edu.cn/).

\section{Plant material}

H. brasiliensis cultivars RY7-33-97 SRJCs were produced through anthers as explants by tissue culture according to Wang et al. (1980) and planted in the Experimental Farm of the Institute of Tropical Bioscience and Biotechnology. Latex (LX), bark (BA), Root (RT), leaf (LF), flower (FL), anther (AN), callus (CA), somatic embryo (SE) and regenerated plant (BRP) were harvested for RNA extraction.

\section{Expression analysis of $H b H M T s$ and $H b H D M s$}

Isolation of latex RNA was performed according to Tang et al. (2007), and RNA from other tissues was isolated using the RNAprep pure plant kit (TIANGEN, China). Quantitative PCR (qPCR) was performed according to Li et al. (2017). qPCR was performed with the primers listed in Table S2. An actin gene (GenBank HQ260674) was used as internal control. Three independent biological replicates were assayed. Gene expression levels were calculated by the $2^{-\Delta \Delta \mathrm{Ct}}$ method. A heat map was created using $\log _{2}$ based on the average of values from three qPCR data.

\section{Expression profiles of $H b H M T s$ and $H b H D M s$ in latex of SRJCs and DCs}

The transcriptome data of latex from SRJCs and DCs were obtained from a previously study (Li et al., 2016). The Reads Per Kb per Million Reads Mapped (RPKM) value was used to analyze the expression profiles of HbHMTs and $H b H D M s$ in latex of SRJCs and DCs. A heat map was created using $\log _{2}[\mathrm{RPKM}]$.

\section{Data archiving statement}

Nucleotide sequences of 47 HMTs and 25 HDMs were deposited with the GenBank XM_021786768.1, XM_021834736.1, XM_021834736.1, XM_021828898.1, XM_021806158.1, XM_021782931.1, XM_021803290.1, XM_021812039.1, XM_021824874.1, XM_021800084.1, XM_021834835.1, XM_021800066.1, XM_021818539.1, XM_021795250.1, XM_021821496.1, XM_021794700.1, 
XM_021786520.1, XM_021827306.1, XM_021817790.1, XM_021837061.1, XM_021820973.1, XM_021807740.1, XM_021817272.1, XM_021817249.1, XM_021817902.1, XM_021827573.1, XM_021827163.1, XM_021781563.1, XM_021787829.1, XM_021781563.1, XM_021792442.1, XM_021785572.1, XM_021781562.1, XM_021790643.1, XM_021783766.1, XM_021798535.1, XM_021814753.1, XM_021779747.1, XM_021789206.1, XM_021802565.1, XM_021802564.1, XM_021790468.1, XM_021815283.1, XM_021790438.1, XM_021789001.1, XM_021795575.1, XM_021812048.1, XM_021790481.1, XM_021835117.1, XM_021784706.1, XM_021803998.1, XM_021789490.1, XM_021750304.1, XM_021816041.1, XM_021829524.1, XM_021790903.1, XM_021781966.1, XM_021821775.1, XM_021809017.1, XM_021832519.1, XM_021806151.1, XM_021802187.1, XM_021811847.1, XM_021807326.1, XM_021800564.1, XM_021794618.1, XM_021780908.1, XM_021810275.1, XM_021813550.1, XM_021810520.1, XM_021794939.1, XM_021825435.1.

\section{Results}

\section{Identification of $H b H M T s$ and $H b H D M s$}

A total of 72 putative genes, including 47 HbHMTs and $25 \mathrm{HbHDMs}$, were identified in Hevea brasiliensis. $H b H M T$ s were designated as HbSDG1 - HbSDG38, and HbPRM1 - HbPRM9. HbHDMs were named as HbJMJ1 HbJMJ20, and HbLSD1 - HbLSD5. The open reading fra-

mes of the 72 predicted genes ranged from $822 \mathrm{bp}$ (HbSDG35) to $4353 \mathrm{bp}$ (HbLSD5) in length (Table S3). The number of exons of the 72 predicted genes ranged from 1 (HbSDG3, 4, 7, 18, 30 and HbLSD3) to 28 (HbJMJ6).

\section{The conserved domains and phylogenetic analysis of HbHMTs and HbHDMs}

The putative HbHMTs were classified as 9 HbPRMs, and 38 HbSDGs. The HbHDMs were 5 HbLSDs and 20 HbJMJs, according to the previous study in Solanum lycopersicum and Citrus sinensis (Aiese Cigliano et al., 2013; Xu et al., 2015). For HbHMTs, all of the 38 HbSDGs were characterized by a conserved SET domain (PF00856) and were grouped into seven classes. In detail, HbSDG9, 15, 21, 22, and 23, which have a conserved SANT domain (SM00717) and CXC domain (SM001114), belong to class I. Class II consisted of seven HbSDGs, including HbSDG1, $16,19,32-34,37$, and contain a conserved AWS domain (SM00570) and Post-SET domain (SM00508), respectively. Class III was comprised of four members (HbSDG 20, 24, 25, 26), which have a Post-SET domain (SM00508), PHD domain (PF00628), and a PWWP domain (SM00293). Class IV included HbSDG27 and 28, which have an N-terminal PHD domain. Nine HbSDGs (HbSDG2-8, 10, 11) belonged to class $\mathrm{V}$ and contained a Pre-SET domain (PF05033), Post-SET domain (SM00508), and a SRA-YDG domain (IPR003105). Eight HbSDGs belonged to class VI /VII (Figure 1A). With re-
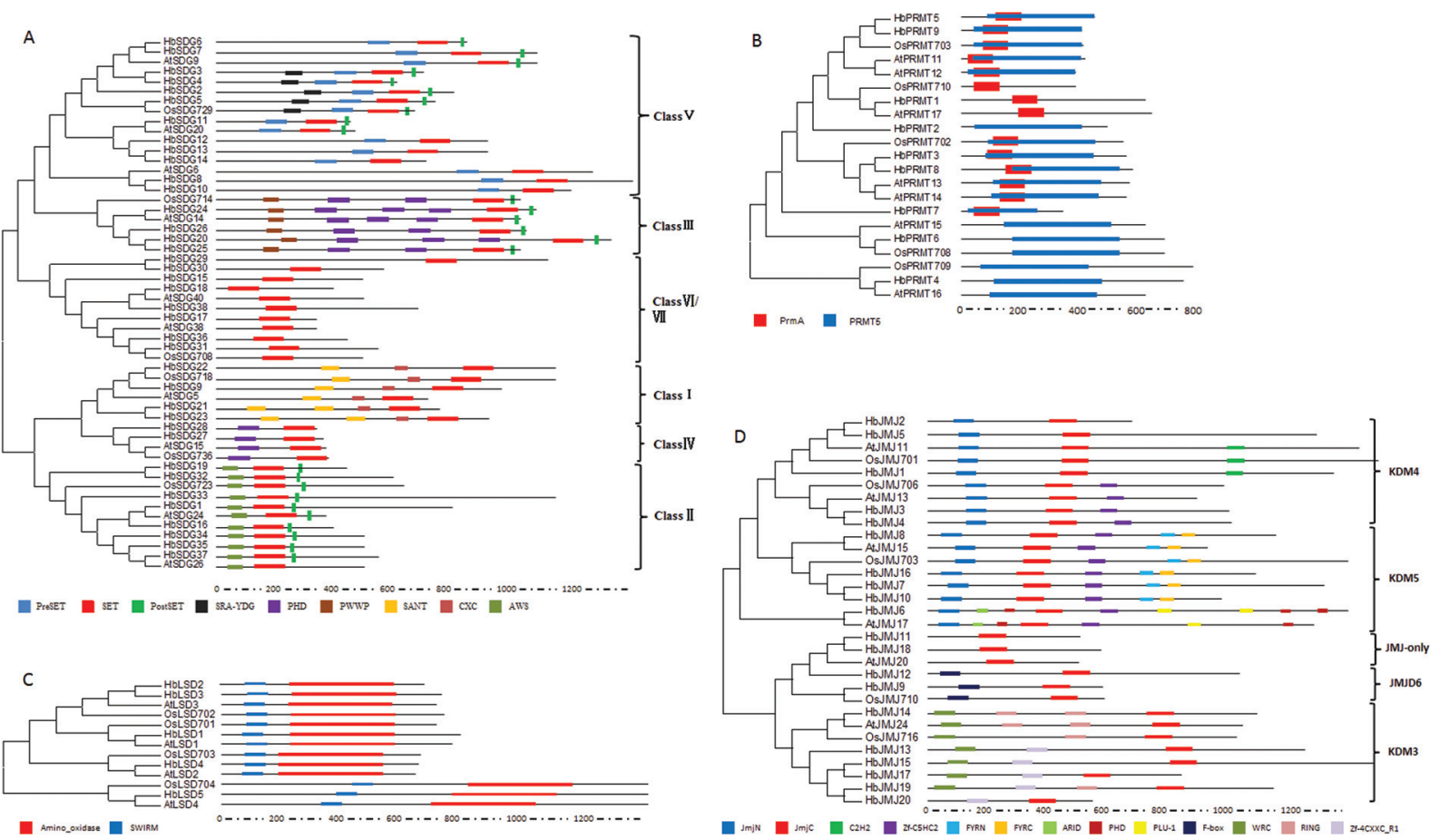

Figure 1 - The conserved domains and phylogenetic analysis of HbHMTs and HbHDMs. (A) The conserved domains and phylogenetic analysis of HbSDGs. (B) The conserved domains and phylogenetic analysis of HbPRMTs. (C) The conserved domains and phylogenetic analysis of HbLSDs. (D) The conserved domains and phylogenetic analysis of HbJMJs. The accession numbers of HMTs and HDMs from Arabidopsis and rice employed for the phylogenetic tree analysis are given in Table S1. 
gard to HbPRMTs, nine predicted HbPRMs proteins were characterized by a PRMT5 domain (PF05185) and categorized into two classes. HbPRMT 1, 3-5, 7-9 belonged to class I, which contains a PrmA domain (PF06325), and HbPRMT4 and 6 were clustered into class II (Figure 1B). For HbHDMs, all of the five HbLSDs have a conserved domain and an amino oxidase domain (PF01593) and SWIRM (PF04433) (Figure 1C). All of the 20 HbJMJs have a conserved JmjC domain (PF02373). The JMJ family is divided into five classes, including JMJ-only, KDM3, KDM4, KDM5, and JMJD6, according to the previous study by Lu et al. (2011). The JMJ-only class consisted of HbJMJ11 and 18, which only contain the conserved JmjC domain. KDM3 class included seven members (HbJMJ3, 14-15, 17, 19 and HbJMJ20), characterized by a ring finger domain (SM000184). The KDM4 group HbJMJs were classified into two main subgroups. Subgroup I contains a ZnF_C2H2 domain (SM000355), while subgroup II has a zf-C5HC2 domain (PF02928) at the C-terminus. The class KDM5 comprises five members (HbJMJ6-8, 10, 16) and is characterized by a JmjN domain (PF02375) and zf-C5HC2 domain (PF02928), respectively. Additionally, two members (HbJMJ9 and HbJMJ12) belonged to the JMJD6 class, which contains an N-terminal F-box domain (PF00646) (Figure 1D).

\section{Expression of $H b H M T s$ and $H b H D M s$}

The expression of HbHMTs and HbHDMs was investigated in LX, BA, RT, LF, and FL. As shown in Figure 2A and Figure $3 \mathrm{~A}$, all these genes were differentially expressed either in terms of their expression patterns or their transcript level. Among the HbHMTs, HbPRMT18 and $H b S D G 25$ were highly expressed in all tested tissues, while $H b S D G 17,26,36$, and 38 had low expression in five tested tissues. $H b P R M T 1,3,7$ and $H b S D G 7,11$ showed a high expression level in LX, while HbPRMT4 and $H b S D G 1,12$, 35 were highly expressed in LF. HbPRMT4 and HbSDG1, 12,36 had high expression in FL. Some HbHMTs, such as $H b P R M T 7$ and $H b S D G 12,21$ were highly expressed in RT. HbPRMT1 and HbSDG1, 33 showed a high expression

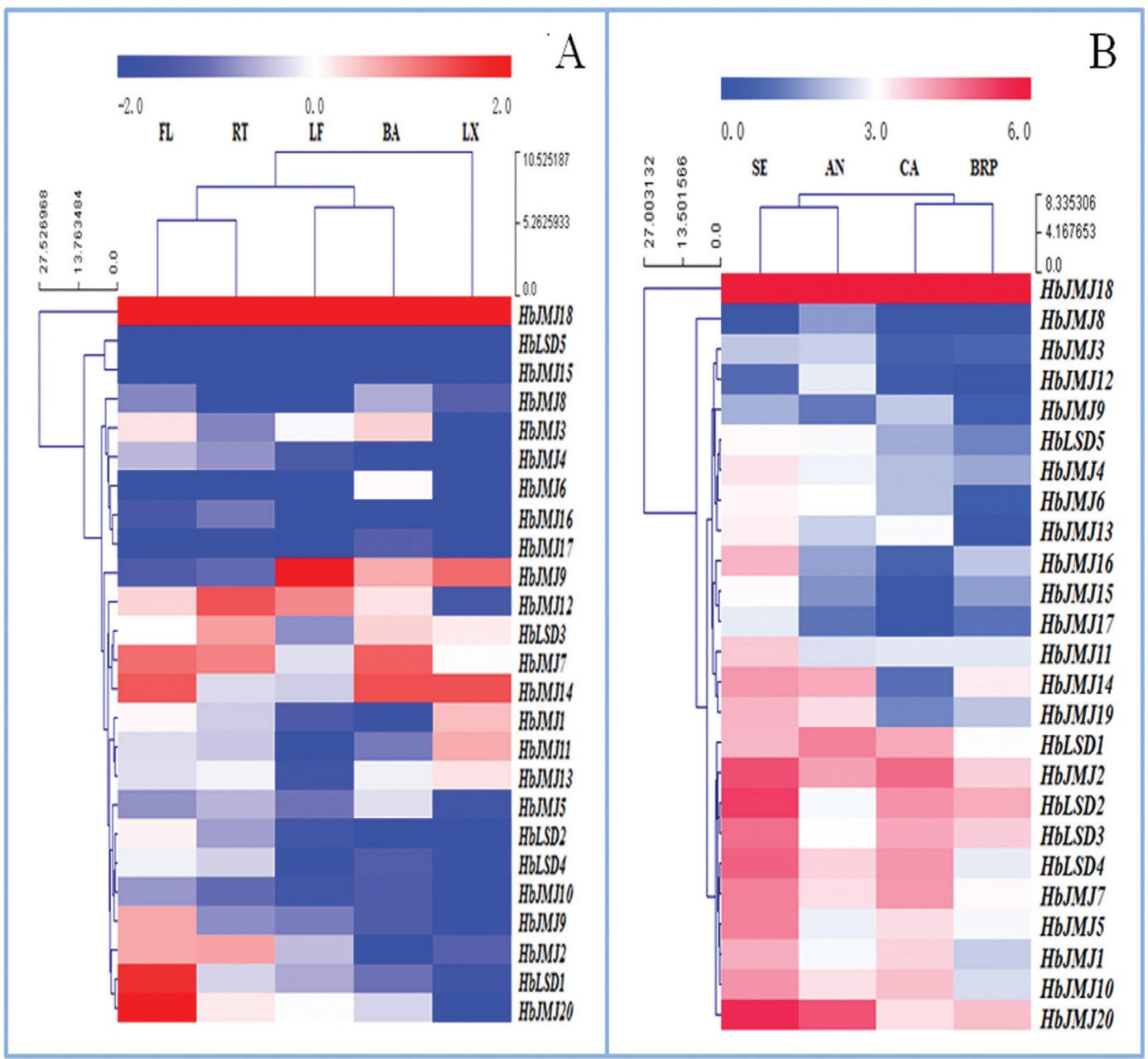

Figure 2 - The expression of HbHMTs in different tissues (A) and during somatic embryogenesis of rubber tree (B). The heat maps were created using $\log _{2}$ based values from three replicates of real-time RT-PCR data. The scale represents the relative signal intensity values. RT, Root; BA, bark; LF, leaf; FL, flowers; LX, latex; AN, Anther; CA, callus; SE, somatic embryo, BRP, bud of regenerated plant. 
level in BA (Figure 2A). Among the HbHDMs, HbJMJ18 was highly expression in all tested tissues, while $H b J M J 15$ and $H b L S D 5$ had low expression in five tested tissues. $H b J M J 1,14$ showed a high expression level in LX, while HbJMJ9, 12 were expressed highly in LF. HbJMJ7, 14,18 and HbLSD1 had high expression in FL. HbJMJ7, 12 and HbLSD3 were highly expressed in RT. HbJMJ7, 14 showed a high expression level in BA (Figure 3A).

The expression of HbHMTs and HbHDMs at different phases of somatic embryogenesis

An expression analysis of HbHMTs and HbHDMs was performed to further the understanding of the mechanisms involved in rubber tree somatic embryogenesis. Most of the HbHMTs and HbHDMs had differential expression profiles during somatic embryogenesis. Among the HbHMTs, HbSDG10, 21, 25, 33 showed high expression at different phases of somatic embryogenesis, while HbSDG4, 9, 16, 17, 19, 26, 27, 29 and HbPRMT3, 6, 7, 12 were with low expression at different phases of somatic embryogenesis. HbSDG10,14, 20, 21, 33 and HbPRMT4 were expressed highly in AN, HbSDG14, 20, 21, 22, 23, 33, 35 and HbPRMT1 showed a high expression level in CA. HbSDG1, 7, 10, 13, 14, 18, 19, 21, 22, 23, 35, and HbPRMT1, 8 were expressed highly in SE. HbSDG10, 21, 25, 33 were expressed highly in BRP (Figure 2B). Among the $H b H D M s, H b J M J 2,18,20$ were with high expression at different phases of somatic embryogenesis, while $H b J M J 3$, 8, 9, 12, 17 and HbLSD5 were with low expression at different phases of somatic embryogenesis. Additionally, HbJMJ7 and HbLSD1, 2, 3, 4, showed a high expression level in CA .HbLSD2, 3 were highly expressed in BRP. HbJMJ2, 14, 20 and HbLSD1 were highly expressed in AN. HbJMJ5, 7, 11, 16, 20, and HbLSD2, 3, 4 were highly expressed in SE. HbLSD2, 3 showed a high expression level in BRP (Figure 3B).

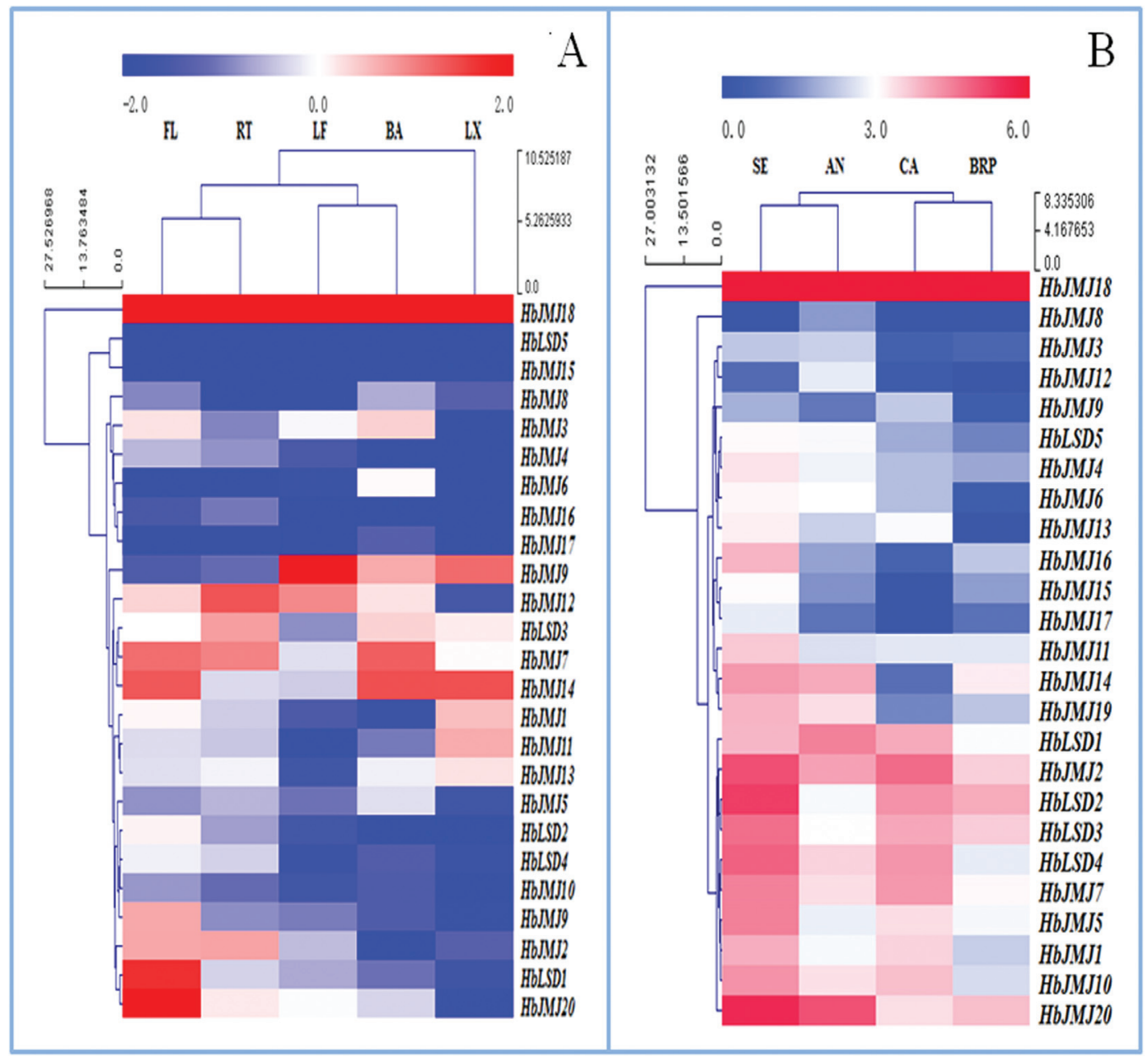

Figure 3 - The expression of $H b H D M s$ in different tissues (A) and during somatic embryogenesis of rubber tree (B). The heat maps were created using $\log _{2}$ based values from three replicates of real-time data. The scale represents the relative signal intensity values. RT, Root; BA, bark; LF, leaf; FL, flowers; LX, latex; AN, Anther; CA, callus; SE, somatic embryo, BRP, bud of regenerated plant. 


\section{Expression profiles of $H b H M T s$ and $H b H D M s$ in latex compatomg SRJCs and DCs}

The expression patterns of HbHMTs and HbHDMs in latex were analyzed comparing SRJCs and its DCs. Eleven HbHMTs (HbSDG 1, 7-10, 19, 22, 27, 33, 34 and HbPRMT6) and five HbHDMs (HbJMJ3, 4, 6, 12, 18 and $H b L S D 5)$ had differential expression profiles in latex between SRJCs and DCs. Among these 11 differentially expressed HbHMTs, HbSDG1, 7, 9, 19, 22 were up-regulated in SRJCs, while HbSDG8, 10, 27, 33, 34 and HbPRMT6 were up-regulated in DCs (Figure 4A).Among the five $H b H D M s$ differentially expressed in latex between SRJCs and DCs, HbJMJ3, 6, 12 were up-regulated in SRJCs, while $H b J M J$ 4, 18 and HbLSD5 were up-regulated in DCs (Figure 4B).

\section{Discussion}

Plant regeneration via somatic embryogenesis provokes many epigenetics changes including histone modifi-
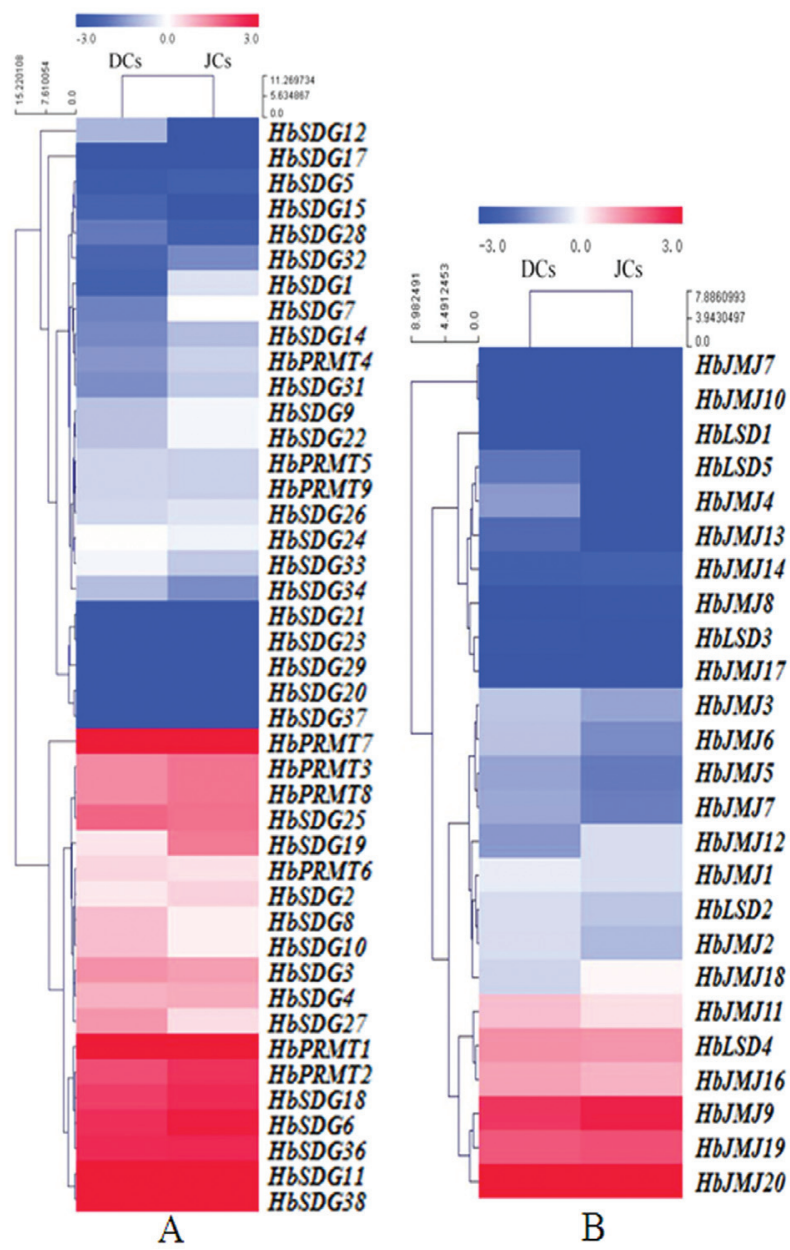

Figure 4 - Expression profiles of $H b H M T s$ and $H b H D M s$ in latex. (A) Heatmap of HbHMTs. (B) Heatmap of HbHDMs. The heatmaps were created using $\log _{2}$ based RPKM values from the latex transcriptome data of SRJCs and its DCs. The values in red and blue indicate $\log _{2}[\mathrm{RPKM}]$ fold increases and decreases, respectively. cation and DNA methylation (Yakovlev et al., 2016). Histone modification is dynamically regulated during somatic embryogenesis (Pfluger and Wagner, 2007; Eichten et al., 2014; De-la-Peña et al., 2015). The dynamic activity in the modification of histones leads to the modulation of gene expression involved in somatic embryogenesis (Nic-Can et al., 2013). For example, the levels of the histone repressive marks H3K9me 2 and H3K27me3 decrease in C. canephora during the early events of somatic embryogenesis, and these events were correlated with the beginning of the expression of genes involved in the somatic embryogenesis process. This indicates that the histone modification regulated changes in transcriptional program of the somatic cells before and during the development of somatic embryos (Nic-Can et al., 2013). The H3K9me 2 mark has also been involved in embryo cell differentiation and heterochromatization events during microspore embryogenesis in B. napus (Rodríguez-Sanz et al., 2014). In addition, the expression patterns of several genes related to histone modification have been studied during the somatic embryogenesis process of $Q$. suber (Pérez et al., 2015). It was found that $Q S H D A 19$ decreases as soon as the callus begins its differentiation, followed by a steady increase from immature cotyledonary embryo to an embryo with fully differentiated cotyledons. On the other hand, a transient decrease in QSHDA6, QSPICKLE, and QSVAL1 gene expression was observed at the transition from callus to the end of the mature embryo.

Histone methylation is generally associated with regulating dynamic changes from heterochromatin formation to transcriptional regulation (Kouzarides, 2002). The methylation of histones is involved in repressing or activating gene expression (Lusser et al., 2002). The methylation and demethylation of histones modifications was shown to be associated the with chromatin state in modulating plant growth and development (Berr et al., 2011; Xu et al., 2015). Here, histone methylation modifiers involved in histone methylation/demethylation have been identified in Hevea brasiliensis. Four gene groups ( $H b S D G, H b P R M, H b L S D$, and $H b J M J$ ) containing 72 genes were characterized in Hevea brasiliensis. The numbers of these groups in Hevea brasiliensis are close to those in Citrus sinensis and Arabidopsis. HbHMTs and HbHDMs have 47 and 25 members, respectively, and the corresponding AtHMTs and AtHDMs families contain 48 and 25 members (Gu et al., 2016), CsHMTs and CsHDMs families contain 47 and 23 members (Xu et al., 2015).

The expressions profiles of all HbHMTs and $H b H D M s$ were analyzed in rubber tree tissues. The differential expression of HbHMTs and HbHDMs in different rubber tree tissues indicates a broad role for some $H b H M T s$ and HbHDMs. The highly expressed HbHMTs and $H b H D M s$ or differentially expressed HbHMTs and $H b H D M s$ in different tissues may play an important role in 
rubber tree growth and development. It will be of interest to elucidate the functions of $H b H M T s$ and $H b H D M s$.

During plant somatic embryogenesis, plant growth regulators may contribute to induce epigenetic modifications (Miguel and Marum, 2011; Machczynska et al., 2015). The modification of histones leads to the modulation of the expression of genes involved in the somatic embryogenesis process (De-la-Peña et al., 2015). In this study, several genes encoding histone methylation modifiers were shown to be differentially expressed in latex comparing SRJCs and DCs, including eleven HbHMTS (HbSDG 1 , 7-10, 19, 22, 27, 33, 34 and HbPRMT6) and five HbHDMs ( $H b J M J 3,4,6,12,18$ and $H b L S D 5$ ). Latex is the cytoplasm of laticifer cells in $H$. brasiliensis (Chow et al., 2007). Laticifers in the bark of the rubber tree are specific for rubber biosynthesis (Hao and $\mathrm{Wu}, 2000$ ). Histone methylation modifications, thus, may provide new insights into the molecular mechanism associated with high yield in SRJCs.

\section{Acknowledgments}

This study was supported by the National Natural Science Foundation of China (No. 31670611), the National Key R\&D Program of China (No. 2018YFD1000502) and the Central Public-Interest Scientific Institution Basal Research Fund for the Chinese Academy of Tropical Agricultural Sciences (No. 1630022018018).

\section{Conflict of Interest}

The authors declare that they have no conflict of interest.

\section{Author Contributions}

SQP conceived the study. HLL, DG, YW, and JHZ performed the experiments and carried out the analysis. HLL and SQP designed the experiments and wrote the manuscript.

\section{References}

Ahmad A and Cao X (2012) Plant PRMTs broaden the scope of arginine methylation. J Genet Genomics 39:195-208.

Aiese Cigliano R, Sanseverino W, Cremona G, Ercolano MR, Conicella C and Consiglio FM (2013) Genome-wide analysis of histone modifiers in tomato: gaining an insight into their developmental roles. BMC Genomics 14:57.

Backhaus RA (1985) Rubber formation in plants. Israel J Bot 34:283-293.

Bailey TL, Johnson J, Grant CE and Noble WS (2015) The MEME Suite. Nucleic Acids Res 43:39-49.

Berr A, McCallum EJ, Alioua A, Heintz D, Heitz T and Shen WH (2010) Arabidopsis histone methyltransferase SET DOMAIN GROUP8 mediates induction of the jasmonate/ethylene pathway genes in plant defense response to necrotrophic fungi. Plant Physiol 154:1403-1414.
Berr A, Shafiq S and Shen WH (2011) Histone modifications in transcriptional activation during plant development. Biochim Biophys Acta 1809:567-576.

Carron MP and Enjalric F (1982) Studies on vegetative micropropagation of Hevea brasiliensis by somatic embryogenesis and in vitro microcutting. In: Fujiwara A (ed) Plant Tissue Culture. Maruzen, Tokyo, pp 751-752.

Chandrashekar TR, Mydin KK, Alice J, Varghese YA and Saraswathyamma CK (1997) Intraclonal variability for yield in rubber (Hevea brasiliensis). Ind J Nat Rubber Res 10:43-47.

Chang B, Chen Y, Zhao Y and Bruick RK (2007) JMJD6 is a histone arginine demethylase. Science 318:444-447.

Cartagena JA, Matsunaga S, Seki M, Kurihara D, Yokoyama M, Shinozaki K, Fujimoto S, Azumi Y, Uchiyama S and Fukui S (2008) The Arabidopsis SDG4 contributes to the regulation of pollen tube growth by methylation of histone $\mathrm{H} 3$ lysines 4 and 36 in mature pollen. Dev Biol 315:355-368.

Chen XT, Wang ZY, Wu HD and Zhang XJ (2002) A new planting material of Hevea brasiliensis self-rooting juvenile-type clone. Chinese J Trop Crops 23:192-193.

Cho JN, Ryu JY, Jeong YM, Park J, Song JJ, Amasino RM, Noh B and Noh YS (2012) Control of seed germination by lightinduced histone arginine demethylation activity. Dev Cell 22:736-748.

Chow KS, Wan KL, Isa MN, Bahari A, Tan SH, Harikrishna K and Yeang HY (2007) Insights into rubber biosynthesis from transcriptome analysis of Hevea brasiliensis latex. J Exp Bot 58:2429-2440.

Clément-Demange A, Priyadarshan PM, Hoa TTT and Venkatachalam P (2007) Hevea rubber breeding and genetics. In: Janick J (ed) Plant Breeding Reviews. Wiley and Sons, Hoboken, pp 177-281.

De-la-Peña C, Nic-Can GI, Galaz-Ávalos RM, Avilez-Montalvo R and Loyola-Vargas VM (2015) The role of chromatin modifications in somatic embryogenesis in plants. Front Plant Sci 6:635.

Eichten SR, Schmitz RJ and Springer NM (2014) Epigenetics: beyond chromatin modifications and complex genetic regulation. Plant Physiol 165:933-947.

Gan ES, Xu Y, Wong JY, Goh JG, Sun B, Wee WY, Huang J and Ito $T$ (2014) Jumonji demethylases moderate precocious flowering at elevated temperature via regulation of FLC in Arabidopsis. Nat Commun 5:5098.

Grini PE, Thorstensen T, Alm V, Vizcay-Barrena G, Windju SS, Jorstad TS, Wilson ZA and Aalen RB (2009) The ASH1 HOMOLOG 2 (ASHH2) histone H3 methyltransferase is required for ovule and anther development in Arabidopsis. PLoS One 4:e7817.

Gu T, Han Y, Huang R, McAvoy RJ and Li Y (2016) Identification and characterization of histone lysine methylation modifiers in Fragaria vesca. Sci Rep 6:23581.

Hao BZ and $\mathrm{Wu}$ JL (2000) Laticifer differentiation in Hevea brasiliensis: Induction by exogenous jasmonic acid and linolenic acid. Ann Bot 85:37-843.

Hua YW, Huang TD and Huang S (2010) Micropropagation of self-rooting juvenile clones by secondary somatic embryogenesis in Hevea brasiliensis. Plant Breed 129:202-207.

Jenuwein T and Allis CD (2001) Translating the histone code. Science 293:1074-1080. 
Jones MA and Harmer S (2011) JMJD5 functions in concert with TOC1 in the Arabidopsis circadian system. Plant Signal Behav 6:445-448.

Klose RJ and Zhang Y (2007) Regulation of histone methylation by demethylimination and demethylation. Nat Rev Mol Cell Biol 8:307-818.

Kouzarides T (2002) Histone methylation in transcriptional control. Curr Opin Genet Dev 12:198-209.

Li HL, Guo D and Peng SQ (2014) Differential gene expression profiles in latex from Hevea brasiliensis between selfrooting juvenile and donor clones. Plant Growth Regul 74:65-71.

Li HL, Guo D and Peng SQ (2015) Changes in DNA methylation during somatic embryogenesis of Hevea brasiliensis. J Trop Subtrop Bot 23:527-533.

Li HL, Guo D, Zhu JH, Wang Y, Chen XT and Peng SQ (2016) Comparative transcriptome analysis of latex reveals molecular mechanisms underlying increased rubber yield in Hevea brasiliensis self-rooting juvenile clones. Front Plant Sci 7:1204.

Li HL, Guo D, Zhu JH, Wang Y and Peng SQ (2017) Identification and expression analysis of genes involved in histone acetylation in Hevea brasiliensis. Tree Genet. Genomes 13:98.

Li J, Yu C, Wu H, Luo Z, Ouyang B, Cui L, Zhang J and Ye Z (2015) Knock down of a JmjC domain-containing gene JMJ524 confers altered gibberellin responses by transcriptional regulation of GRAS protein lacking the DELLA domain genes in tomato. J Exp Bot 66:1413-1426.

Litt M, Qiu Y and Huang S (2009) Histone arginine methylations: Their roles in chromatin dynamics and transcriptional regulation. Biosci Rep 29:131-141.

Liu B, Wei G, Shi J, Jin J, Shen T, Ni T, Shen WH, Yu Y and Dong A (2016) SET DOMAIN GROUP 708, a histone H3 lysine 36-specific methyltransferase, controls flowering time in rice (Oryza sativa). New Phytol 210:577-588.

Liu C, Lu F, Cui X and Cao X (2010) Histone methylation in higher plants. Annu Rev Plant Biol 61:395-420.

Liu K, Yu Y, Dong A and Shen WH (2017) SET DOMAIN GROUP 701 encodes a H3K4-methytransferase and regulates multiple key processes of rice plant development. New Phytol 215:609-623.

Liu SQ, Yuan XH, Huang X and Xu LY (1985) Comparative studies on yield and properties of juvenile type- and its mature type clones. Trop Crop Res 3:1-5.

Liu X, Zhou C, Zhao Y, Zhou S, Wang W and Zhou DX (2014) The rice enhancer of zeste $[\mathrm{E}(\mathrm{z})]$ genes SDG711 and SDG718 are respectively involved in long day and short day signaling to mediate the accurate photoperiod control of flowering time. Front Plant Sci 5:591.

Liu X, Zhou S, Wang W, Ye Y, Zhao Y, Xu Q, Zhou C, Tan F, Cheng S and Zhou DX (2015) Regulation of histone methylation and reprogramming of gene expression in the rice inflorescence meristem. Plant Cell 27:1428-1444.

Lu SX, Knowles SM, Webb CJ, Celaya RB, Cha C, Siu JP and Tobin EM (2011) The JumonjiC domain-containing protein JMJ30 regulates period length in the Arabidopsis circadian clock. Plant Physiol 155:906-915.

Lusser A (2002) Acetylated, methylated, remodeled: chromatin states for gene regulation. Curr Opin Plant Biol 5:437-443.
Machczynska J, Zimny J and Bednarek PT (2015) Tissue culture-induced genetic and epigenetic variation in triticale (Triticosecale spp.Wittmack ex A. Camus 1927) regenerants. Plant Mol Biol 89:279-292.

Miguel C and Marum L (2011) An epigenetic view of plant cells cultured in vitro: Somaclonal variation and beyond. J Exp Bot 62:3713-3725.

Mitchell A, Chang HY, Daugherty L, Fraser M, Hunter S, Lopez R, McAnulla C, McMenamin C, Nuka G, Pesseat S et al. (2014) The InterPro protein families database: The classification resource after 15 years. Nucleic Acids Res 43:213-221.

Nic-Can GI, López-Torres A, Barredo-Pool F, Wrobel K, Loyola-Vargas VM, Rojas-Herrera R and De-la-Peña C (2013) New insights into somatic embryogenesis: leafy cotyledon1, baby boom 1 and WUSCHEL-related homeobox 4 are epigenetically regulated in Coffea canephora. PLoS One 8:e72160.

Ng DW, Wang T, Chandrasekharan MB, Aramayo R, Kertbundit $\mathrm{S}$ and Hall TC (2007) Plant SET domain-containing proteins: structure, function and regulation. Biochim Biophys Acta 1769:316-329.

Pérez M, Cañal M and Toorop P (2015) Expression analysis of epigenetic and abscisic acid-related genes during maturation of Quercus suber somatic embryos. Plant Cell Tissue Organ Cult 121:353-366.

Pfluger J and Wagner D (2007) Histone modifications and dynamic regulation of genome accessibility in plants. Curr Opin Plant Biol 10:645-652.

Rahman AYA, Usharraj AO, Misra BB, Thottathil GP, Jayasekaran K, Feng Y, Hou S, Ong SY, Ng FL, Lee LS et al. (2013) Draft genome sequence of the rubber tree (Hevea brasiliensis). BMC Genomics 14:75.

Rice JC, Briggs SD, Ueberheide B, Barber CM, Shabanowitz J, Hunt DF, Shinkai Y and Allis CD (2003) Histone methyltransferases direct different degrees of methylation to define distinct chromatin domains. Mol Cell 12:1591-1598.

Rodríguez-Sanz H, Moreno-Romero J, Solís MT, Köhler C, Risueño MC and Testillano PS (2014) Changes in histone methylation and acetylation during microspore reprogramming to embryogenesis occur concomitantly with BnHKMT and BnHAT expression and are associated with cell totipotency, proliferation, and differentiation in Brassica napus. Cytogenet Genome Res 143:209-218.

Shen Y, Silva N, Audonnet L, Servet C. Wei W and Zhou DX (2014) Over-expression of histoneH3K4 demethylase gene JMJ15 enhances salt tolerance in Arabidopsis. Front Plant Sci 5:290.

Shi Y, Lan F, Matson C, Mulligan P, Whetstine JR, Cole PA, Casero RA and Shi Y (2004) Histone demethylation mediated by the nuclear amine oxidase homolog LSD1. Cell 119:941-953.

Sui PF, Jin J, Ye S, Mu C, Gao J, Feng HY, Shen WH, Yu Y and Dong A (2012) H3K36 methylation is critical for brassinosteroid-regulated plant growth and development in rice. Plant J 70:340-347.

Tang C, Qi J, Li H, Zhang C and Wang Y (2007) A convenient and efficient protocol for isolating high-quality RNA from latex of Hevea brasiliensis (para rubber tree). J Biochem Biophys Methods 70:749-754. 
Tang C, Yang M, Fang Y, Luo Y, Gao S, Xiao X, An Z, Zhou B, Zhang B, Tan X et al. (2016) The rubber tree genome reveals new insights into rubber production and species adaptation. Nat Plants 2:16073.

Thorstensen T, Grini PE and Aalen RB (2011) SET domain proteins in plant development. Biochem Biophys Acta 1809:407-420.

Tsukada Y, Fang J, Erdjument-Bromage H, Warren ME, Borchers $\mathrm{CH}$, Tempst $\mathrm{P}$ and Zhang Y (2006) Histone demethylation by a family of JmjC domain containing proteins. Nature 439:811-816.

van Dijk K, Ding Y, Malkaram S, Riethoven JJ, Liu R, Yang J, Laczko P, Chen H, Xia Y, Ladunga I et al. (2010) Dynamic changes in genome-wide histone $\mathrm{H} 3$ lysine 4 methylation patterns in response to dehydration stress in Arabidopsis thaliana. BMC Plant Biol 10:238.

Wang Z, Zeng X, Chen C, Wu H, Li Q, Fan G and Lu W (1980) Induction of rubber plantlets from anther of Hevea brasiliensis in vitro. Chin J Trop Crops 1:25-26.

Xu J, Xu H, Liu Y, Wang X, Xu Q and Deng X (2015) Genome-wide identification of sweet orange (Citrus sinensis) histone modification gene families and their expression analysis during the fruit development and fruit-blue mold infection process. Front Plant Sci 6:607.

Yakovlev IA, Carneros E, Lee Y, Olsen JE and Fossdal CG (2016) Transcriptional profiling of epigenetic regulators in somatic embryos during temperature induced formation of an epigenetic memory in Norway spruce. Planta 243:1237-49.

Yang SQ and Mo YY (1994) Some physiological propeptries of latex from somatic plants of Hevea brasiliensis. Chin J Trop Crops 15:13-20.
Yuan XH, Yang SQ, Xu LY, Wu JL and Hao BZ (1998) Characteristics related to higher rubber yield of Hevea brasiliensis juvenile-type clone G11. J Rubber Res 1:125-132.

Zhao M, Yang S, Liu X and Wu K (2015) Arabidopsis histone demethylases LDL1 and LDL2 control primary seed dormancy by regulating DELAY OF GERMINATION 1 and ABA signaling-related genes. Front Plant Sci 6:159.

\section{Internet Resources}

ClustalX2, http://www. clustal.org/.

GSDS Software, http://gsds.cbi. pku.edu.cn/.

Meme Suite, http://meme-suite.org/tools/meme.

Pfam, http://pfam.sanger. ac.uk/search.

SMART, http://smart.embl-heidelberg.de/.

\section{Supplementary material}

The following online material is available for this article:

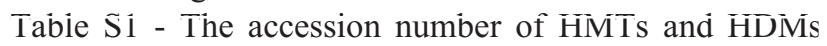
from Arabidopsis and rice.

Table S2 - Overview of the primer sequences for real time PCR.

Table S3 - Overview of the detected histone modification genes in Hevea brasiliensis.

Associate Editor: Adriana S. Hemerly

License information: This is an open-access article distributed under the terms of the Creative Commons Attribution License (type CC-BY), which permits unrestricted use, distribution and reproduction in any medium, provided the original article is properly cited. 